\title{
Flexicurity - Ein Konzept für das Arbeitsrecht der Zukunft?
}

Die rechtspolitische Diskussion hat auf nationaler und auf europäischer Ebene das Arbeitsrecht im Visier. Die Union und die FDP verlangen einschneidende Änderungen sowohl im Individualarbeitsrecht, insbesondere beim Kündigungsschutz, wie auch im Tarifvertragsrecht und bei der Mitbestimmung. Auch die Europäische Kommission hat sich mit dem Grünbuch „Ein modernes Arbeitsrecht für die Herausforderungen des 21. Jahrhunderts“ über ein Flexicurity-Konzept in die Diskussion eingebracht. Wie sind diese Vorschläge zu bewerten? Brauchen wir mehr Deregulierung? Oder gibt es Bereiche, in denen zugunsten der sozialen Sicherheit eine andere Regulierung oder gar mehr Regulierung notwendig sind? Und welche Chancen hätte ein solches Flexicurity-Konzept angesichts der jüngeren Erfahrungen mit Gesetzesreformen und angesichts der Richtung, in die rechtspolitische Debatten in Deutschland gehen?

\section{Das Grünbuch der Europäischen Kommission}

Mit dem Grünbuch zum Arbeitsrecht soll unter dem Begriff Flexicurity eine Diskussion zur Reform des Arbeitsrechts angestoßen werden. Darin stellt die Europäische Kommission Fragen und erwägt Modelle, die die Flexibilität auf den Arbeitsmärkten und Beschäftigungssicherheit in ein ausgewogenes Verhältnis bringen sollen. Das Arbeitsrecht und die geltenden Tarifverträge würden dem Bedürfnis nach flexibleren Beschäftigungsverhältnissen vonseiten der Unternehmen und größtmöglicher Sicherheit vonseiten der Beschäftigten nicht mehr gerecht. Die Reform solle einen Arbeitsmarkt fördern, der gerechter, reaktionsfähiger und integrativer sei. Die Debatten darum sollen bis zum Ende des Jahres 2007 $\mathrm{zu}$ gemeinsamen Grundsätzen für den Flexicurity-Ansatz in den Staaten der Europäischen Union führen.

Flexicurity ist ein vielversprechender Ansatz, geht er doch davon aus, zwei bislang eher als Gegensätze begriffene und jedenfalls isoliert verfolgte Ziele auszubalancieren. Flexicurity könnte das deutsche Arbeitsrecht durchaus zukunftsfähig machen. Der Vorstoß der Europäischen Kommission könnte allerdings auch dazu verwendet werden - und das Grünbuch selbst nährt in erheblichem Ausmaß diesen Verdacht -, dass die „Kluft zwischen Insidern und Outsidern" (Kommission der Europäischen Gemeinschaften 2006, S. 8, 11) lediglich durch Abbau von Schutzrechten der Beschäftigten verkleinert wird, Flexibilisierung also leichter durchgesetzt wird, die
Stärkung sozialer Sicherheit aber papierenes Versprechen bleibt. Es stellen sich deshalb drei Fragen: zum einen nach dem konkreten Inhalt eines Flexicurity-Konzeptes, zum anderen, ob und wo es Defizite an Flexibilität und Sicherheit im Arbeitsrecht in Deutschland gibt und schließlich, welche Regulierungen zu einem Flexicurity-Recht führen können, das diesen Namen verdient. Dabei müssen die Rechtsmaterien zusammengedacht werden, also neben dem Arbeitsrecht müssen das Sozialrecht und das Steuerrecht gleichermaßen berücksichtigt werden.

\section{Inhalte eines Flexicurity- Konzeptes}

Die Europäische Kommission hat im Grünbuch keine konkreten Vorgaben für ein Flexicurity-Konzept gemacht, in der Mitteilung dazu vom 27.6.2007 (Kommission der Europäischen Gemeinschaften 2007) aber die nach seiner Sicht notwendigen Komponenten beschrieben: flexible und sichere vertragliche Vereinbarungen, umfassende Strategien des lebenslangen Lernens, wirksame aktive arbeitsmarktpolitische Maßnahmen und moderne Systeme der sozialen Sicherheit. Die Strategien und Maßnahmen müssten allerdings den äußerst unterschiedlichen Situationen in den einzelnen EU-Mitgliedstaaten Rechnung tragen.

Für die Bundesrepublik Deutschland soll im Folgenden ein Flexicurity-Konzept zur Diskussion gestellt werden, das - dem Ansatz entsprechend - über die Grenzen des Arbeitsrechts hinausgeht (vgl. dazu auch Kronauer/Linne 2005):
(1) Beschäftigungsversicherung und Absicherung von Übergängen: Sollen die Menschen Mobilität akzeptieren und sie von sich aus zeigen, dürfen flexible und unterschiedliche Formen des Erwerbs sowie Erwerbsbrüche nicht zu Lücken der sozialen Sicherheit, nämlich in der Versicherungsbiografie und im sozialen Schutz führen. Die Versicherungspflicht muss mit dem Ziel der bevölkerungsweiten Abdeckung für Basisrisiken formuliert werden; ihr Ausmaß muss allen Bürgerinnen und Bürgern über den Lebensverlauf zumindest eigenständige Alterssicherungsansprüche in Höhe des soziokulturellen Minimums garantieren. Dazu gehört ein breiterer $\mathrm{Zu}$ gang zu allen sozialen Sicherungssystemen, und zwar unabhängig vom Status als Arbeitnehmer. Der Übergang in die Selbstständigkeit oder in abhängige Arbeit muss versicherungspraktisch bedeutungslos sein. Die Art der Beschäftigung - als Arbeitnehmer, per Dienst- oder Werkvertrag - darf weder beim Beschäftiger noch beim Beschäftigten zur Vermeidung oder Verminderung von Versicherungspflichten führen.

(2) Inklusion in „Gute Arbeit“": Eine Politik der Inklusion muss sich der Frage widmen, wie Personen in Erwerbsarbeit hineinfinden und gehalten werden können, und zwar in nicht-prekäre Erwerbsarbeit. Niemand darf dauerhaft in Arbeitslosigkeit und in prekären oder unfreiwillig in atypischen Arbeitsverhältnissen verbleiben müs-

Heide Pfarr, Prof. Dr., Geschäftsführerin der Hans-Böckler-Stiftung und Wissenschaftliche Direktorin des WSI. e-mail: heide-pfarr@boeckler.de 
sen. Auch hier sind die Übergänge zu sichern. Die staatliche Bevorzugung von Mini- und Midijobs muss beseitigt, Leiharbeit auf Aushilfsjobs begrenzt werden. Für Arbeitsentgelte müssen Untergrenzen gesetzlich fixiert werden.

(3) Interne Flexibilität und Employability: Die Unternehmen sind aufgefordert, Formen der internen Flexibilität denen der externen vorzuziehen. Dies verlangt von ihnen einen vorausschauenden Umgang mit Aus- und Weiterbildung, mit lebenslangem Lernen, mit alters- und alternsgerechten Arbeitsbedingungen und mit arbeitsplatzsichernden Arbeitszeitanpassungen. Dazu müssen auch zwischenbetriebliche Arbeitsmärkte und Konstruktionen überbetrieblicher Arbeitsplatzsicherung entwickelt und vom Staat unterstützt werden. Weiterbildung wird gebraucht, um die Beschäftigungsfähigkeit aufrechtzuerhalten oder herzustellen. Hier geht es um geregelte Ansprüche auf Zeit und finanzielle Unterstützung.

(4) Lebenslauforientierte Anpassung von Beschäftigungszeiten: Die in der europäischen Beschäftigungsstrategie fixierten Ziele, die Erwerbsquoten von Frauen und älteren Menschen zu steigern, wie auch das Leitbild des „adult workers“ erfordern Antworten auf die Frage, von wem, wie und wann jene Arbeiten erbracht werden sollen, die nicht ausschließlich über den Markt zu organisieren sind: beispielsweise die Erziehung von Kindern und die Pflege älterer Angehöriger. Dafür müssen garantierte Optionen gewährt werden, also Rechte auf Zeit, die es Erwerbstätigen ermöglichen, das Ausmaß der Erwerbstätigkeit jeweils ihren individuellen Bedürfnissen und Lebensverhältnissen anzupassen, Verhältnissen, die sich im Lebensverlauf wandeln können. Dabei ist die entscheidende Frage, wie die mit Erwerbsunterbrechungen oder -einschränkungen entstehenden Einkommensausfälle finanziell abgesichert werden sollen. Die Überantwortung allein an die Betroffenen bzw. deren Familien reicht nicht aus. Denn es gibt erhebliche Differenzen: Menschen haben ungleiche Bedürfnisse nach erwerbsfreier oder erwerbsgeminderter Zeit und dies zu unterschiedlichen Zeiten. Bei einigen häufen sich z. B. Fürsorgearbeiten, während sie bei anderen bedeutungslos sind. Die ungleiche Betroffenheit der Geschlechter ist offensichtlich. Ein sozialer Ausgleich ist deshalb unver- zichtbar. Das gilt jedenfalls für bestimmte Lebensphasen und Sachverhalte, die als gesellschaftlich nützlich und unterstützenswert erscheinen. Eine lebenslaufzentrierte Sozialpolitik muss über kollektive Systeme für eine finanzielle Abfederung sorgen, die ausreichend intertemporal und interpersonell verteilt (Klammer 2005).

\section{Regulierungsanforderun- gen für ein Flexicurity- Konzept}

Die Anforderungen an ein bundesdeutsches Flexicurity-Konzept sind also hoch. Selbstverständlich ist es möglich und wohl auch angemessen, nicht den großen Wurf auf einmal $\mathrm{zu}$ wagen, sondern sich in Schritten dem Ziel zu nähern nach dem Beispiel jener europäischen Länder, die bereits Ansätze von Flexicurity umgesetzt haben (Kommission der Europäischen Gemeinschaften 2007, S. 23ff.). Aber auch dann gilt: Der Gesetzgeber stünde vor einer gewaltigen Aufgabe, wenn er diesen Anspruch ernst nehmen und in Normen gießen will. Dabei gibt es eine bedeutsame politische und regulatorische Schieflage: Deregulierungen sind gesetzestechnisch einfach, Flexibilisierungen und die Beschneidung sozialen Schutzes finden in den Medien vielfach Befürworter. Für ihre Durchsetzung in der Praxis werden schon die begünstigten Unternehmen sorgen. Ganz anders sieht es mit neuen Instrumenten der sozialen Sicherheit aus. Hier wird es jeweils heftigen und medial unterstützten Widerstand aus der Wirtschaft geben. Und mehr noch: Es verlangt regulatorischen Einfallsreichtum, Individuen mit real durchsetzbaren Rechten auszustatten. Was nutzt zum Beispiel einer Arbeitnehmerin ein gesetzlicher Anspruch auf berufliche Weiterbildung, welche Chancen der Verwirklichung hat sie? Würde er nicht dasselbe Schicksal haben wie die Ansprüche nach den Weiterbildungsgesetzen der Länder, von denen gut $1 \%$ der Beschäftigten Gebrauch machen (Brandner 2007)? Anders als bei der Flexibilisierung durch Deregulierung bedarf es hier innovativer Förderung und mehrerer handelnder Akteure.

Der staatliche Gesetzgeber müsste die Instrumente der sozialen Sicherung und die individuellen Ansprüche im Gesetz zwar absichern, für die Durchsetzung in der Praxis aber bedürfte es kollektiver Vereinbarungen der Tarif- und Betriebsparteien. Die Bedingungen für interne Flexibilitäten, für die Weiterbildung mit lebenslangem Lernen, für die Entwicklung alternsgerechter Arbeitsbedingungen und für Arbeitszeitanpassungen sind unterschiedlich je nach Branche, Unternehmensgröße, Arbeitskräftepotenzial. Dasselbe gilt für die Organisation zwischenbetrieblicher Arbeitsmärkte und überbetriebliche Arbeitsplatzsicherung. Angemessene Regulierungsinstrumente sind hier Kollektivvereinbarungen, insbesondere Tarifverträge, von denen Sachnähe und Interessenrepräsentanz erwartet werden müssten.

Von Erwartungen ist mit Bedacht die Rede. Denn so selbstverständlich jahrzehntelang in der Bundesrepublik das Verweisen auf und das Vertrauen in Tarifverträge berechtigt war, so sehr hat sich die Tariflandschaft inzwischen geändert - Dieterich hat (in diesem Heft) darauf hingewiesen. Sie ist zersplittert, und nicht nur in Ostdeutschland gibt es eine Fülle von Tarifverträgen, deren Inhalt mit Unterbietung gesetzlicher oder tariflicher Standards abschließend beschrieben ist. Auch die DGB-Gewerkschaften werden so öfter in eine Tarifpolitik gezwungen, deren Ergebnisse sie selbst nicht wirklich vertreten können. Die vielfältigen Bündnisse für Arbeit, die Ausweitung von Leiharbeit, von atypischen und prekären Beschäftigungsverhältnissen belegen, in welchem Maße Betriebsräte unter Druck geraten sind. Sie alle aber - durchsetzungsfähige Tarifvertragsparteien und eine starke betriebliche Interessenvertretung - werden gebraucht, soll ein Flexicurity-Konzept, das diesen Namen verdient, mit Leben erfüllt werden.

\section{Gesetzesreformen und rechtspolitische Debatten zum Arbeitsrecht}

Will man die Chancen eines FlexicurityKonzepts in der Rechtspolitik Deutschlands einschätzen, kann es hilfreich sein, zu fragen, in welche Richtung die Antworten gegangen sind, die die Gesetzgebung und die rechtspolitische Debatte in Deutschland bislang gefunden haben (Seifert 2006). 


\subsection{EINORDNUNG DER GESETZES- REFORMEN}

Dieterich hat (in diesem Heft) die Entwicklungen im Individualarbeitsrecht und im Tarifrecht dargestellt, die schon am Maßstab des hergebrachten deutschen Arbeitsrechts zu Funktionsstörungen und Problemen geführt haben. Der Gesetzgeber hat hier nicht (oder nur vorläufig wie bei der Scheinselbstständigkeit) nach Abhilfe gesucht. Auffällig ist demgegenüber, dass in den letzten 20 Jahren die Rechtsreformen vor allem in Richtung auf mehr Flexibilität vorangetrieben wurden und das Stichwort Deregulierung die Agenda bestimmte und das von politisch unterschiedlich zusammengesetzten Regierungskoalitionen. Der Gesetzgeber lockerte etwa die Einschränkungen für Befristungen und bei der Leiharbeit, verkürzte den Kündigungsschutz, ließ im Arbeitszeitgesetz verlängerte Arbeitszeiten zu und förderte in hohem $\mathrm{Maß}$ atypische und prekäre Beschäftigung durch die Bedingungen für geringfügige Beschäftigung sowie die Veränderung der Rahmenbedingungen für Transfereinkommen in den Hartz-Gesetzen.

Hingegen stießen auf vehementen Widerstand (vor allem der Wirtschaft und der BDA) jene Gesetzesreformen der letzten 20 Jahre, die durchaus der Stärkung der sozialen Sicherheit zuzuordnen sind, beispielsweise der Anspruch auf Absenkung der Arbeitszeit nach dem Teilzeit- und Befristungsgesetz, die Veränderungen bei den Regelungen zur Elternzeit und schließlich das Entsendegesetz und dessen Ausweitungen. Andere Reformen sind durch Europäisches Recht erzwungen worden und spiegeln nicht den Willen der Mehrheit des Deutschen Bundestages: So das Verbot der Benachteiligung von Teilzeitbeschäftigten und die anderen Diskriminierungsverbote bis hin zum Allgemeinen Gleichbehandlungsgesetz.

Bewertet man diese Gesetzesreformen am Maßstab von Flexicurity, ergibt sich ein gemischtes Bild mit überwiegend negativen Effekten und damit eine pessimistische Prognose für dieses Konzept.

Es gibt Stimmen, die behaupten, die Hartz-Gesetze hätten den jetzigen Abbau der Arbeitslosigkeit positiv beeinflusst. Auf die freiwillige Mobilität von Beschäftigten aber haben sie sich mit Sicherheit negativ ausgewirkt. Die Angst davor, womöglich unter den Bedingungen von Hartz IV leben zu müssen, bringt Arbeitnehmer eher dazu, sich am Bestehenden festzuklammern. Die anderen deregulierenden Gesetzesreformen haben, wie empirisch belegt, keinerlei beschäftigungsfördernde Wirkungen gezeitigt, obwohl das erste entsprechende Gesetz 1985 sogar dieses Ziel im Namen trug. Die Deregulierungen haben aber nicht nur einschneidende Folgen für die Arbeitslosen und die Ränder des Arbeitsmarktes, sondern wirken bis weit hinein in die Kernbelegschaften. Sie haben deren Vertrauen in die soziale Sicherung erschüttert und schließlich auch negativ auf das Lohnniveau Einfluss genommen.

Die Reformen, die der Stärkung der sozialen Sicherung dienen, greifen den Gesichtspunkt der solidarischen finanziellen Abfederung lebenslauforientierter Bedürfnisse nur andeutungsweise auf. Sie werden insgesamt konterkariert durch die staatliche Subventionierung von Mini- und Midijobs, die unvermeidlich zu Armut im Alter führen und die Sozialsysteme gefährden. Darüber hinaus ist zu fragen, ob die Durchsetzbarkeit der Ansprüche ausreichend abgesichert ist (vgl. den Beitrag von Kocher in diesem Heft).

\subsection{DIE RECHTSPOLITISCHE DEBATTE}

\section{TARIFRECHT}

Die stärksten Flexibilisierungen hat es allerdings nicht durch den Gesetzgeber, sondern durch die im Beitrag von Dieterich (in diesem Heft) beschriebene veränderte Tarifpraxis der Tarifvertragsparteien gegeben. Eine betriebsnahe und differenzierte Umsetzung eines Flexicurity-Konzepts in kollektivrechtliche Vereinbarungen mit Verpflichtungen und umsetzbaren Ansprüchen wäre so bei ihnen in guten Händen. Doch die rechtspolitische Debatte geht in die entgegengesetzte Richtung. Es mag Ausdruck eines gewandelten Verhältnisses zum Sinn und Zweck des kollektiven Arbeitsrechts - und auch der sozialen Sicherheit - sein, dass in Rechtswissenschaft und Politik ganz offen und intensiv nach Wegen gesucht wird, wie Unternehmen tarifvertragliche Bindungen gänzlich abschütteln können, und zwar ohne die Lästigkeit von Verhandlungen mit den Tarifvertragsparteien. Bedeutsam sind vor allem zwei Konzepte, die dieses Ziel ganz konsequent verfolgen: Eine modifizierte Interpretation des Günstigkeitsprinzips und die Abdingung des Tarifvorrangs. Wie sind sie rechtlich einzuschätzen, welche
Folgen hätte eine derartige Reform und was sagt deren anhaltende Debatte aus über die Chancen, Flexicurity umzusetzen?

(1) Günstigkeitsprinzip und zwingende Wirkung des Tarifvertrages: Im wissenschaftlichen Schrifttum wird die Auffassung vertreten, es bedürfe gar keiner Gesetzesänderung, um weitere Flexibilisierungen durchzusetzen. Der Vorrang der Tarifverträge vor betrieblichen Regelungen lasse sich durch Änderung der bisherigen Rechtsprechung zum Günstigkeitsprinzip vermeiden (Buchner 1999, S. 901f. mwN). Nach dem Tarifvertragsgesetz sind Vereinbarungen, die vom Tarifvertrag abweichen, zulässig, soweit sie durch den Tarifvertrag selbst gestattet sind oder eine Änderung zugunsten des Arbeitnehmers enthalten. Aber was ist "günstiger"? Dazu sagt das Gesetz selbst nichts. Das BAG hat aus der Funktion der Tarifautonomie abgeleitet, dass ein Sachgruppenvergleich vorzunehmen ist. Im Gegensatz dazu wollen einige Autoren im Rahmen des Günstigkeitsvergleichs beschäftigungssichernde Zusagen berücksichtigen und zur Kompensation untertariflicher Leistungen zulassen. Das Bundesarbeitsgericht wird aufgefordert, seine Maßstäbe für den Günstigkeitsvergleich zu korrigieren. Die Sicherung von Arbeitsplätzen sei für die betroffenen Arbeitnehmer allemal wichtiger als Mindestlöhne und Arbeitszeiten. Wenn man das bei der Bewertung der „Bündnisse für Arbeit“ angemessen berücksichtige, fielen alle Bedenken in sich zusammen.

Eine solche Auslegung des Günstigkeitsprinzips erhielte aber dadurch eine systemwidrige, ja sogar systemsprengende Wirkung, wie schon Dieterich (2002, S. 14f.) überzeugend nachgewiesen hat. Der Sinn dieses Prinzips besteht darin, den einzelnen Arbeitnehmer von kollektiven Zwängen freizustellen, soweit er auf kollektivrechtlichem Schutz nicht angewiesen ist. Die zwingende Wirkung des Tarifvertrags als Auffangnetz wird dadurch aber nicht infrage gestellt. Sie ist sogar die Voraussetzung des Günstigkeitsprinzips. Bei Verhandlungen über Arbeitsverträge sollen die tariflichen Standards stets die Basis bilden. Wenn diese Basis durch vertragliche Einheitsregelungen mit dem Segen des Betriebsrats beiseite geschoben werden könnte, verlagere sich nur die Regelungskompetenz auf ein anderes Kollektiv. Aus dem Günstigkeitsprinzip würde ein Ablösungs- 
prinzip. Das sei nach geltendem Recht nicht möglich.

(2) Gesetzesinitiativen zum Vorrang betrieblicher Regelungen: Diese Erkenntnis hat zum Ruf nach dem Gesetzgeber geführt. Der Vorrang der Tarifverträge vor betrieblichen Regelungen soll generell beseitigt werden. Darauf zielen Gesetzesinitiativen von CDU/CSU und FDP (CDU/CSUFraktion 2003; FDP-Fraktion 2003).

Solche Vorschläge verkennen das Schutzkonzept der Tarifautonomie grundlegend. Gerade die existenzielle Angewiesenheit auf Arbeitsmöglichkeiten begründet das Schutzbedürfnis, das durch kollektive Interessenwahrung aufgefangen werden soll und nur mit Hilfe zwingender Regelungen abzusichern ist. Könnte diese Wirkung genau dann generell durchbrochen werden, wenn sich das Arbeitsplatzrisiko aktualisiert, würden Tarifverträge zu bloßen Richtlinien für „Schönwetterzeiten“. Tarifpolitisch und organisationspolitisch hätte eine solche Abwertung der Tarifautonomie zerstörerische Vorwirkungen. Der ganze Aufwand tarifpolitischer Konzeptionen und Verhandlungen hätte kaum noch Sinn; schon entwickelte Flexibilisierungsmodelle liefen leer und verlören ihre differenzierende Steuerungsfunktion. Die Arbeitgeberverbände könnten immerhin auf OT-Mitgliedschaften verzichten. ${ }^{1}$ Den Gewerkschaften hingegen würde die Möglichkeit genommen, auf die Arbeits- und Wirtschaftsbedingungen maßgebenden Einfluss zu nehmen. Auf der Betriebsebene müssten sie zudem mit einer gestärkten, aber beitragsfreien Zwangsrepräsentation - dem Betriebsrat - konkurrieren. All das hätte mit der Koalitionsfreiheit, die Art. 9 III GG gewährleisten will, kaum noch Ähnlichkeit (Dieterich 2007, Art. 9 Rn. 63ff.). $\mathrm{Ob}$ es den Interessen der Unternehmen wirklich dienen könnte, erscheint höchst zweifelhaft. Dezentrale Regelungsmodelle sind für betriebsnahe Flexicurity-Konzepte überaus sinnvoll, dezentrale Verteilungskämpfe sind es mit Sicherheit nicht.

\section{KÜNDIGUNGSSCHUTZ}

Obwohl die Behauptung, der deutsche Kündigungsschutz sei beschäftigungs- feindlich, empirisch nicht belegt werden kann, fordern politische Parteien (Union, FDP) wie auch Stimmen in der Rechtsund in der Wirtschaftswissenschaft weiterhin eine Lockerung oder gar gänzliche Beseitigung des Kündigungsschutzes (Sachverständigenrat 2007, S. 415ff.; Donges et al. 2004). Das Projekt REGAM des WSI und weitere Forschungen der Hans-Böckler-Stiftung haben sich damit ausführlich auseinandergesetzt. Deren Ergebnisse sind so eindeutig, dass es hier einer erneuten Widerlegung nicht mehr bedarf (Pfarr et al. $2005 \mathrm{mwN}$ ).

Der Maßstab des Flexicurity-Konzepts verlangt, dass Reformforderungen an den Kündigungsschutz auch daran gemessen werden, ob sie die interne Flexibilität gegenüber der externen - also der Exit-Option - bevorzugen. Die bloße Verkürzung des Kündigungsschutzes läuft dem zuwider, denn sie erleichtert ja die Beendigung des Arbeitsverhältnisses. Die EU-Kommission betont im Übrigen, dass ein erfolgreiches Flexicurity-Konzept auf Vertrauen in das System basiert. Gleichzeitig zeigt sie allerdings eine besondere Vorliebe für Regulierungsmodelle mit geringem Kündigungsschutz. Hierbei ist zu bedenken, dass die Bedürfnisse der Menschen in der EU und damit die Bedingungen, unter denen Vertrauen entsteht oder gefährdet wird, durchaus unterschiedlich sind. In Deutschland ist - wie Untersuchungen ergeben haben (Krause 2006, S. 105ff.; dies. 2007) ein verhältnismäßig gut ausgebauter Kündigungsschutz wesentlicher Bestandteil des Vertrauens auf soziale Sicherheit. Wer da unter Verweis auf Dänemark, wo längere Betriebsdauer nicht als erstrebenswert gilt, den Kündigungsschutz in Deutschland angreift, beseitigt bei den Beschäftigten die notwendige Vertrauensbasis in ein Flexicurity-Konzept.

\section{Fazit}

Dies alles kann nicht hoffnungsfroh stimmen. Die bisherigen Gesetzesreformen unterschiedlicher politischer Koalitionen entsprechen ganz und gar nicht den Vorstellungen, die mit einem Flexicurity-Konzept verbunden sind. Der heftige und aggressive Widerstand seitens der Wirtschaft gegenüber den zaghaftesten Anstrengungen, durch neue Gesetze etwas mehr soziale Sicherheit zu geben, lassen ahnen, wie viel Augenmaß und Entschlossenheit der Gesetzgeber benötigt, wenn er hier anpacken will. Die andauernde Diskussion um den Kündigungsschutz im konservativen Teil der großen Koalition wirkt negativ auf Mobilitätsbereitschaft und das Vertrauen in die soziale Sicherung. Art und Ausmaß der aktuellen Debatten um die Reformen der sozialen Sicherungssysteme lassen zweifeln, ob der bundesdeutsche Gesetzgeber in der derzeitigen Koalition willens und mehr noch: imstande wäre, das erforderliche Ausmaß sowohl an Flexibilität wie an sozialer Sicherheit durch den weitgehenden Umbau dieser Systeme zu gewährleisten. Die tarifrechtlichen Reformvorschläge der Union und der FDP zeigen, dass diejenigen Akteure, die für ein lebendiges und differenziertes Flexicurity-Konzept unverzichtbar sind - die Gewerkschaften als Tarifvertragsparteien - entmachtet und an den Rand gedrängt werden sollen. Von diesen beiden Parteien wird die Beschwörung der Tarifautonomie nur noch verwandt, um einen gesetzlichen Mindestlohn durchaus Bestandteil eines FlexicurityKonzepts - zu verhindern.

Flexicurity ist ein unterstützenswertes Regulationsmodell. Das Konzept bietet die Chance, Flexibilität und Sicherheit in ein ausgewogenes Verhältnis zu bringen. Allerdings: Nicht alles, was unter dem Etikett Flexicurity diskutiert wird, verdient diesen Namen. Angesichts der Debattenkultur und der politischen Mehrheitsverhältnisse in Deutschland sind die gegenwärtigen Vorraussetzungen für die Umsetzung von Flexicurity eher schlecht. Der Verdacht liegt nahe, die Diskussion um Flexicurity könnte zur Verschleierung und Rechtfertigung weiterer Deregulierungsvorhaben dienen. Ein guter Ansatz, für den zu streiten sich lohnt, würde damit vertan.

OT-Mitgliedschaft bezeichnet die Mitgliedschaft eines Arbeitgebers in einem Arbeitsgeberverband, ohne dass eine Tarifbindung entsteht. 
Brandner, K. (2007): Politik für Arbeitnehmerinnen und Arbeitnehmer. Für eine lebendige Weiterbildungskultur, download unter: http://www.klausbrandner.de/.net//themen/10096/209601.html Buchner, H. (1999): Der Unterlassungsanspruch der Gewerkschaften Stabilisierung oder Ende des Verbandstarifvertrags?, in: Neue Zeitschrift für Arbeitsrecht 17, S. 897-902

CDU/CSU-Fraktion (2003): Entwurf eines Gesetzes zur Modernisierung des Arbeitsrechts (ArbRModG), Bundestagsdrucksache 15/1182 Dieterich, T. (2007): in: Dieterich, T./Müller-Glöge, R./Preis, U./Schaub, G.: Erfurter Kommentar, 7. Aufl., München

Dieterich, T. (2002): Flexibilisiertes Tarifrecht und Grundgesetz, in: Recht der Arbeit 1, S. 1-17

Donges, J./Eeckhoff, W. F./Möschel, W./Neumann, M. (2004): Flexibler Kündigungsschutz am Arbeitsmarkt, Schriftenreihe Stiftung Marktwirtschaft, Bd. 41, Berlin 2004, download unter: http://www.insm.de/ Downloads/PDF_Dateien/Publikationen_Kostenlose_Downloads/ Studie-Kuendigungsschutz.pdf

FDP-Fraktion (2003): Entwurf eines Gesetzes zur Sicherung betrieblicher Bündnisse für Arbeit, Bundestagsdrucksache 15/1225

Klammer, U. (2005): Flexicurity aus der Perspektive des Lebenslaufs, in: Kronauer, M./Linne, G.: Flexicurity. Die Suche nach Sicherheit in der Flexibilität, Berlin 2005, S. 249-273

Kommission der Europäischen Gemeinschaften (2006): Grünbuch „Ein modernes Arbeitsrecht für die Herausforderungen des 21. Jahrhunderts, download unter: http://ec.europa.eu/employment_social/labour_law/ docs/2006/green_paper_de.pdf
Kommission der Europäischen Gemeinschaften (2007): Mitteilung der Kommission an das Europäische Parlament, den Rat, den Europäischen Wirtschafts- und Sozialausschuss und den Ausschuss der Regionen: Gemeinsame Grundsätze für den Flexicurity-Ansatz herausarbeiten: Mehr und bessere Arbeitsplätze durch Flexibilität und Sicherheit, download unter: http://ec.europa.eu/employment_social/employment_strategy/pdf/ flex_comm_de.pdf

Krause, A. (2006): Die Einstellung zum Kündigungsschutz - wie wichtig sind Gerechtigkeitsnormen und Entlassungserfahrungen, in: Struck, O./Stephan, G./Köhler, Ch./Krause, A./Pfeifer, Ch./Sohr, T.: Arbeit und Gerechtigkeit. Entlassungen und Lohnkürzungen im Urteil der Bevölkerung, Wiesbaden

Krause, A. (2007): Kündigungsschutzreformen und Gerechtigkeit - Einstellungen der Erwerbsbevölkerung, in: WSI-Mitteilungen 5, S. 252-258 Kronauer, M./Linne, G. (2005): Flexicurity. Die Suche nach Sicherheit in der Flexibilität, Berlin 2005

Pfarr, H./ Ullmann, K./Bradtke, M./Schneider, J./Kimmich, M./Bothfeld, S. (2005): Der Kündigungsschutz zwischen Wahrnehmung und Wirklichkeit. Betriebliche Erfahrungen mit der Beendigung von Arbeitsverhältnissen, München und Mering Sachverständigenrat zur Begutachtung der gesamtwirtschaftlichen Entwicklung (SVR) (2007): Jahresgutachten 2006/07, Widerstreitende Interessen - Ungenutzte Chancen, Siebtes Kapitel, download unter: http://www.sachverstaendigenrat-wirtschaft.de/download/gutachten/ ga06_vii.pdf Seifert, H. (2006): Was hat die Flexibilisierung des Arbeitsmarktes gebracht?, in: WSI-Mitteilungen 11, S. 601-608 